\title{
Multipoint Vibration Response Prediction under Uncorrelated Multiple Sources Load Based on Elastic-Net Regularization in Frequency Domain
}

\author{
ZhenKai Cui $₫{ }^{1},{ }^{1}$ Cheng Wang $\left(\mathbb{D},{ }^{1}\right.$ Jianwei Chen, ${ }^{2}$ and Ting $\mathrm{He}^{1}$ \\ ${ }^{1}$ College of Computer Science and Technology, Huaqiao University, Xiamen 361021, China \\ ${ }^{2}$ Department of Mathematics and Statistics, San Diego State University, San Diego, CA 92182, USA \\ Correspondence should be addressed to Cheng Wang; wangcheng@hqu.edu.cn
}

Received 18 October 2020; Revised 12 February 2021; Accepted 19 February 2021; Published 3 March 2021

Academic Editor: Matteo Filippi

Copyright (c) 2021 ZhenKai Cui et al. This is an open access article distributed under the Creative Commons Attribution License, which permits unrestricted use, distribution, and reproduction in any medium, provided the original work is properly cited.

In order to solve the problems of large number of conditions at inherent frequencies and low prediction accuracy when using multiple multivariate linear regression methods for vibration response prediction alone, an elastic-net regularization method is proposed. Firstly, a multi-input and multioutput linear regression model of the multipoint frequency domain vibration response is trained using historical data at each frequency point. Secondly, the trained model under each frequency point is improved by the elastic regularization. Finally, the model is used in a working situation. The predicted vibration response on the experimental dataset of cylindrical shell acoustic vibration showed that the improvement of the multivariate regression vibration response prediction model by elastic regularization can better improve the accuracy and reduce the large number of conditions at some frequencies.

\section{Introduction}

Too large structural vibration response is the chief offender of mechanical failure; accurate and effective vibration response prediction is of great significance to aerospace structures, machinery, buildings, roads, and bridges. The vibration of the structure is caused by excitation, and the vibration response prediction of the system is traditionally solved by measuring the load received by the structure and system characteristics, which belongs to the positive problem in vibration theory. According to the known load conditions, the vibration structure is simplified to obtain a solvable mathematical model, and the vibration response of the structure is solved by certain mathematical methods, used as the basis for vibration assessment of the designed structure. This basic analysis process that is still widely used in engineering problems, especially the vibration theory based on the assumption of linear model, has developed to a very mature stage, and many engineering problems can be applied to this theory to get quite satisfactory results.

Vibration response prediction method field can be divided into frequency-domain method and time-domain method. At present, in the frequency domain, the response prediction of the vibration structure of the traditional methods is based on the load as the input, and the main methods are as follows: (1) based on theoretical solution (only adapted to the simple structure) [1], (2) based on the transfer function and experimental measurement methods [2-4], (3) based on function and data-driven model, and (4) based on machine learning methods. $\mathrm{Li}$ and $\mathrm{Du}$ [5] proposed a response prediction method based on frequency response functions, but this method based on similar transfer functions is prone to matrix inverse pathological problems. It often encounters the ill-defined problems of coefficient matrix solution and singular value decomposition problems $[6,7]$. There are many problems to be solved by regression analysis, but there are few problems to be solved by multiple regression for vibration response prediction in signal processing. Liu mentioned the mathematical modeling approach of multiple linear regression [8], and Wang gave the prediction modeling side of multiple linear regression and how to choose the better model [9-11]. For its application, Peng gave a study of power load prediction based on the multiple linear regression model [12]. There are many 
solutions to practical engineering problems through regularization, but their use to improve vibration response prediction models is rare. Liujin [13] proposed a regularization method and its application in model correction. In order to better demonstrate the role of regularization methods, Huyu [14] proposed a regularization parameter selection method based on parsing. Chitra [15] proposed fractional Tikhonov regularization method (FTRM) for linear ill-posed operator equations in the setting of Hilbert scales, and a new parameter choice strategy is being proposed for choosing the regularization parameter in FTRM in the setting of Hilbert scales. WuYang [16] proposed a nonstationary signal inversion based on shaping regularization for random noise attenuation. The main idea of the method is to use the nonstationary prediction operator (NPO) to describe the complex structure and obtain seismic signals using nonstationary signal inversion instead of convolution.

Traditionally, regardless of transfer function methods or multiple linear regression, there is an indeterminacy problem as well as ill-conditioned matrix problem. To solve this problem, this paper compares three regularization methods (Lasso regression, ridge regression, and elastic network) to alleviate these problems.

The research purpose of this study is to forecast response. The main contributions and novelty of this paper are as follows:
(1) Build a multiple regression prediction model of vibration response for linear systems and improve it using elastic-net regularization methods

(2) Experimental validation is carried out by using the elastic network as an example

(3) This study uses regularization to improve the prediction accuracy and the robustness of the model to some extent

\section{Methodology Introduction to Theoretical Foundations}

2.1. Theoretical Basis of Frequency Domain Vibration Response Prediction Method for Linear Systems under Uncorrelated Known Multiple Source Load Conditions. In the multiexcitation and multiresponse system, the vibration response data of the system can be obtained from the excitation, which indicates the multisource load of the system as the input to the system and the response as the output of the system [17], and the transfer function can be used to solve this problem in this model, which is shown in Figure 1.

In this model, with $m$ uncorrelated multisource loads $\left[f_{1}(\omega), f_{2}(\omega), \ldots, f_{i}(\omega) \ldots f_{m}(\omega)\right]$ at frequency $\omega$ as input and $n$ system response data $\left[y_{1}(\omega), y_{2}(\omega), \ldots, y_{j}(\omega) \ldots y_{n}(\omega)\right]$ as output, the relationship between the response and the uncorrelated multisource loads is shown as follows:

$$
\left[\begin{array}{c}
S_{y_{1} y_{1}}(\omega) \\
\vdots \\
S_{y_{j} y_{j}}(\omega) \\
\vdots \\
S_{y_{n} y_{n}}(\omega)
\end{array}\right]=\left[\begin{array}{ccccc}
\left|H_{1,1}(\omega)\right|^{2} & \cdots & \left|H_{1, i}(\omega)\right|^{2} & \cdots & \left|H_{1, m}(\omega)\right|^{2} \\
\vdots & \ddots & \vdots & \ddots & \vdots \\
\left|H_{j, 1}(\omega)\right|^{2} & \cdots & \left|H_{j, i}(\omega)\right|^{2} & \cdots & \left|H_{j, m}(\omega)\right|^{2} \\
\vdots & \ddots & \vdots & \ddots & \vdots \\
\left|H_{n_{1}, 1}(\omega)\right|^{2} & \cdots & \left|H_{n_{1}, i}(\omega)\right|^{2} & \cdots & \left|H_{n_{1}, m}(\omega)\right|^{2}
\end{array}\right]\left[\begin{array}{c}
S_{f_{1} f_{1}}(\omega) \\
\vdots \\
S_{f_{i} f_{i}}(\omega) \\
\vdots \\
S_{f_{m} f_{m}}(\omega)
\end{array}\right]
$$

In equation (1), $\omega$ represents the frequency, $S_{y_{i} y_{i}}(\omega)$ represents the self-power spectrum of the response point $y_{j}(j=1,2,3, \ldots, n), S_{y_{i} y_{i}}(\omega)$ is the self-power spectrum of the uncorrelated multisource load $f_{i}(i=1,2,3 . ., m)$, and $\left|H_{j, i}(\omega)\right|$ is the mode corresponding to the transfer function from the load $f_{i}(\omega)$ to the response $y_{j}(\omega)$ at the corre-
sponding frequency. $\quad \vec{S}_{Y}(\omega)=\left[\begin{array}{c}S_{y_{1} y_{1}}(\omega) \\ \vdots \\ S_{y_{i} y_{i}}(\omega) \\ \vdots \\ S_{y_{n} y_{n}}(\omega)\end{array}\right], B(\omega)=\left[\begin{array}{c}S_{f_{1} f_{1}}(\omega) \\ \vdots \\ S_{f_{j} f_{j}}(\omega) \\ \vdots \\ S_{f_{n} f_{n}}(\omega)\end{array}\right]$.
$\left[\begin{array}{ccccc}\left|H_{1,1}(\omega)\right|^{2} & \cdots & \left|H_{1, i}(\omega)\right|^{2} & \cdots & \left|H_{1, m}(\omega)\right|^{2} \\ \vdots & \ddots & \vdots & \ddots & \vdots \\ \left|H_{j, 1}(\omega)\right|^{2} & & \left|H_{n, 1}(\omega)\right|^{2} & & \left|H_{j, m}(\omega)\right|^{2} \\ \vdots & \ddots & \vdots & \ddots & \vdots \\ \left|H_{n, 1}(\omega)\right|^{2} & \cdots & \left|H_{n, i}(\omega)\right|^{2} & \cdots & \left|H_{n, m}(\omega)\right|^{2}\end{array}\right], \quad \vec{S}_{F}(\omega)=$

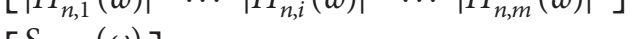$$
\left.\begin{array}{c}
S_{f_{1} f_{1}}(\omega) \\
\vdots \\
S_{f_{j} f_{j}}(\omega) \\
\vdots \\
S_{f_{n} f_{n}}(\omega)
\end{array}\right]
$$ 


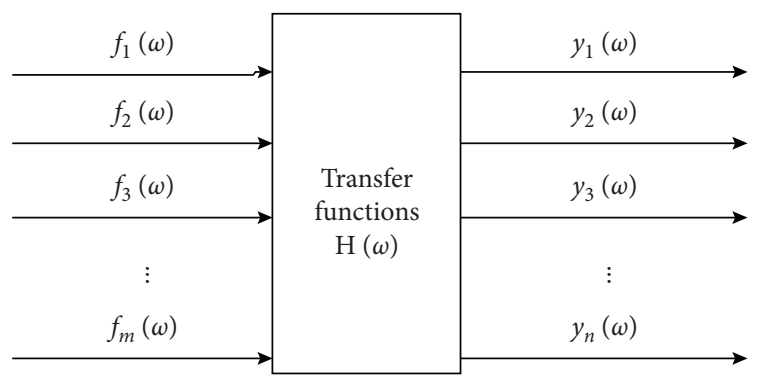

FIGURE 1: Multiple excitations and multiple responses model of linear time-invariant system in the frequency domain. form:

Then, equation (1) may be expressed in the following

$$
\vec{S}_{Y}(\omega)=\mathbf{B}(\omega) \vec{S}_{F}(\omega)
$$

2.2. Multipoint Frequency Domain Vibration Response Prediction Based on Data-Driven and Multiple Linear Regression and Its Existing Problems. In the case where the system frequency domain transfer function $H_{j, i}(\omega)$ in equation (1) is difficult to obtain, data-driven and multiple multivariate regression analysis can be used to regress the system input load to obtain the response value of the system.

The concrete implementation method is as follows: for each frequency point $\omega=1,2, . ., \Omega, \Omega$ is the number of total frequency points, the historical $m$ loads $\left[f_{1}^{p}(\omega), f_{2}^{p}(\omega), \ldots\right.$, $\left.f_{i}^{p}(\omega), \ldots, f_{m}^{p}(\omega)\right]$ of the system are taken as input, the historical $n$ responses $\left[y_{1}^{p}(\omega), y_{2}^{p}(\omega), \ldots, y_{j}^{p}(\omega), \ldots, y_{n}^{p}(\omega)\right]$ are taken as output, and $p=1,2, \ldots, P$, where $P$ is the number of independently repeated experiments. As shown in Figure 2, this $P$ set of historical experimental data is used as multiple-input and multiple-output training data in order to train the multiple multivariate regression analysis model, which is used to train the vibration response prediction model. The trained vibration response prediction model can be used for the working state, load $\left[f_{1}^{\text {work }}(\omega), f_{2}^{\text {work }}(\omega), \ldots\right.$, $\left.f_{j}^{\text {work }}(\omega), \ldots, f_{m}^{\text {work }}(\omega)\right]$ as input of the regression prediction model of the uncorrelated multisource frequency domain load under working state, and the model output $\left[\widehat{y}_{1}^{\text {work }}(\omega), \widehat{y}_{2}^{\text {work }}(\omega), \ldots, \widehat{y}_{j}^{\text {work }}(\omega), \ldots, \widehat{y}_{n}^{\text {work }}(\omega)\right]$ is the predicted result of the uncorrelated multisource frequency domain load. The predicted value can be compared with the measured value $\left[y_{1}^{\text {work }}(\omega), y_{2}^{\text {work }}(\omega), \ldots, y_{j}^{\text {work }}(\omega), \ldots, y_{n}^{\text {work }}\right.$ $(\omega)]$ to quantitatively evaluate the error of the method. The vibration response prediction flow based on multiple linear regression is shown in Figure 2.

However, in practice, regardless of the multiple multivariate linear regression method to establish the model or through the method of transfer function, the established model has a large number of conditions at the inherent frequency and poor recognition accuracy, which will have a great impact on the recognition accuracy. This study will mainly alleviate these problems through various regularization methods.
2.3. Multipoint Frequency Domain Vibration Response Prediction Based on Multiple Multivariate Regularized Linear Regressions. Due to many problems associated with the previously described multiple linear regression vibration response prediction models, the addition of regularization is proposed to alleviate these problems. There are many mainstream regularization methods, and the main ones used in this study are Lasso regression, ridge regression, and elastic network. A schematic diagram of the multipoint frequency domain vibration response prediction by multiple multivariate regularized linear regression is shown in Figure 3.

2.4. Theory of Regularization. Regularization refers to solving the problem of overfitting or large variance errors contained in the model by limiting the size of the hyperparameters; one effective method is the Tikhonov regularization, which aims to solve the following formula [18] to find an approximate solution to the linear problem.

$$
\min \left(\frac{1}{2}\left\|\vec{S}_{Y}(\omega)-A(\omega) \vec{S}_{F}(\omega)\right\|_{2}^{2}+\lambda_{x}(\omega) R(A(\omega))\right) .
$$

where $R(A(\omega))$ is the penalty term that reduces the impact of the indefinite problem. For $\lambda_{x}(x=0,1,2,3)$, the specific function is to balance the relationship between the two objectives. The objective of the first part is to make it fit the training dataset better, and the objective of the second part with the $\lambda$ parameter is to keep the parameter small, while the regularization parameter $\lambda$ needs to control the balance between the two, that is, balancing the objective of fitting the training and the objective of keeping the parameter value small. This is to keep the hypothesis form relatively simple to avoid overfitting. For our vibration response prediction, the very-high-order polynomial we used before to fit would give us a very curved and complex curve function, and now we just use the regularized objective method; then you can get a more appropriate curve, but, instead of a true quadratic function, it is a much smoother and simpler curve. This gives you a better hypothesis for this data. So, in order to make the regularization work well, we should pay attention to some aspects and should go for a good regularization parameter $\lambda$.

Table 1 gives a comparison of the three main regularization methods.

In particular, when the penalty is $(1 / 2)\|A(\omega)\|_{2}^{2}$, it can be called L1 regularization or Lasso [19].

$$
\min \left(\frac{1}{2}\left\|\vec{S}_{Y}(\omega)-A(\omega) \vec{S}_{F}(\omega)\right\|_{2}^{2}\right)+\frac{\lambda_{0}(\omega)}{2}\|A(\omega)\|_{2}^{2},
$$

and, also, when the penalty is $\lambda\|A(\omega)\|_{1}$, it can be called L2 regularization or ridge.

$$
\min \left(\frac{1}{2}\left\|\vec{S}_{Y}(\omega)-A(\omega) \vec{S}_{F}(\omega)\right\|_{2}^{2}\right)+\lambda_{1}(\omega)\|A(\omega)\|_{1} .
$$

The elastic-net regularization is a regularization method combining Lasso with ridge. The naïve elastic-net regularization is defined as 


$$
\begin{gathered}
\text { Training with historical } \\
\text { data } \\
{\left[S_{f_{1} f_{1}}^{p_{1}}(\omega), S_{f_{2} f_{2}}^{p_{2}}(\omega), \ldots, S_{f_{m} f_{m}}^{f_{m}}(\omega)\right]}
\end{gathered}
$$

Enter historical load data that has been measured

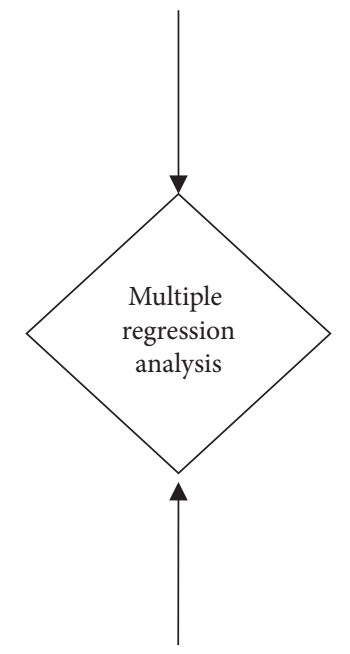

Historical response data measured

$$
\left[S_{y_{1} y_{1}}^{p}(\omega), S_{y_{2} y_{2}}^{p}(\omega), \ldots, S_{y_{m} y_{m}}^{p}(\omega)\right]
$$

Use the trained model for vibration response

prediction under operating conditions

$\left[S_{f_{1} f_{1}}^{\text {work }}(\omega), S_{f_{2} f_{2}}^{\text {work }}(\omega), \ldots, S_{f_{n} f_{n}}^{\text {work }}(\omega)\right]$

Measured load data under actual conditions

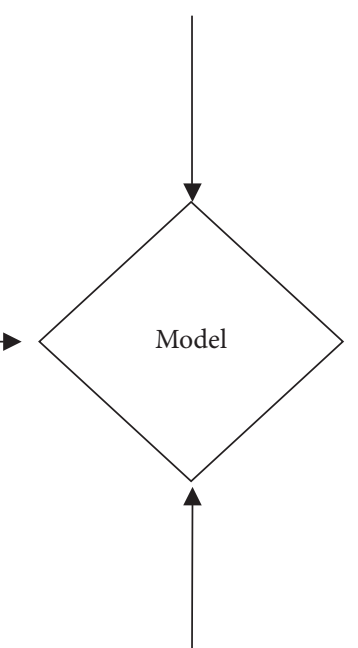

Response data for unmeasured actual operating conditions

$\left[\hat{S}_{y_{1} y_{1}}^{\text {work }}(\omega), \hat{S}_{y_{2} y_{2}}^{\text {work }}(\omega), \ldots, \hat{S}_{y_{n} y_{n}}^{\text {work }}(\omega)\right]$

Figure 2: Flow chart of multiple linear regression prediction.

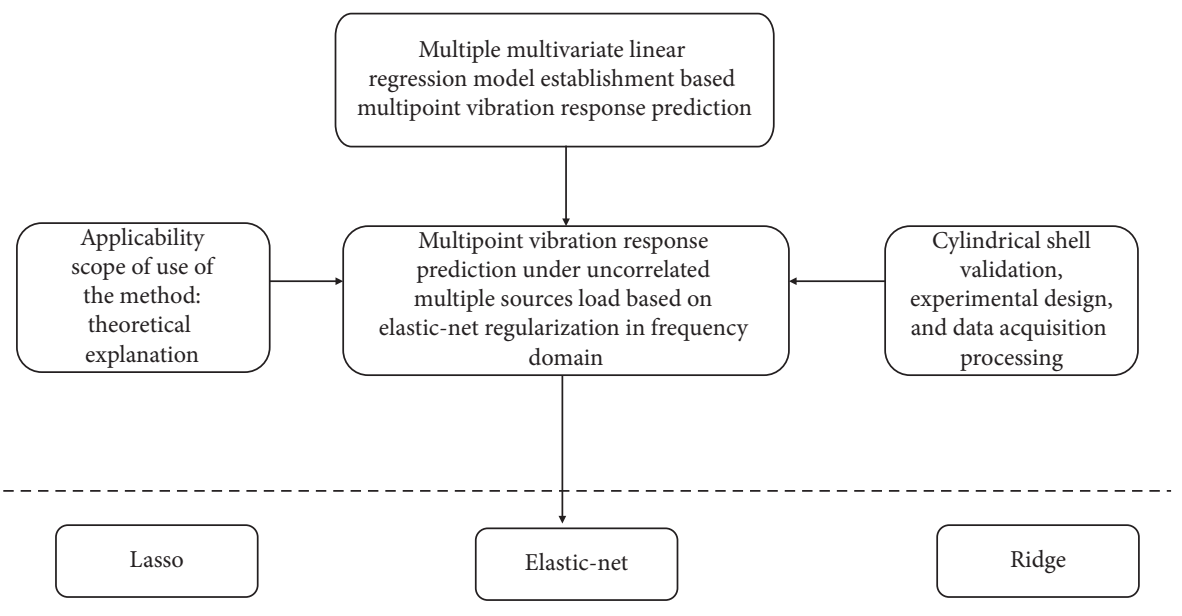

FIGURE 3: Schematic diagram of multivariate linear regression vibration response prediction model based on regularization improvement.

$$
\begin{aligned}
& \min \left(\frac{1}{2}\left\|\vec{S}_{Y}(\omega)-A(\omega) \vec{S}_{F}(\omega)\right\|_{2}^{2}\right)+\lambda_{2}(\omega)\|A(\omega)\|_{1} \\
& \quad+\lambda_{3}(\omega)\|A(\omega)\|_{2}^{2} .
\end{aligned}
$$

where $\lambda_{2}$ and $\lambda_{3} \quad$ are nonnegative, and $\lambda_{2}=a t$ and $\lambda_{3}=(1 / 2) a(1-t)$ are the most commonly used values. In particular, $t$ is the penalty that controls the ratio of Lasso to ridge and $a$ is the constant that multiplies the penalty.

2.5. Comparison of Response Prediction Methods. After experimental validation, we arrive at comparative results on the three current methods. 
TABLE 1: Comparison of regularization methods.

\begin{tabular}{lccc}
\hline Method & Penalty & Advantages & Disadvantages \\
\hline $\begin{array}{l}\text { Lasso } \\
\text { Ridge }\end{array}$ & $\|A(\omega)\|_{1}$ & $\begin{array}{c}\text { This method has the ability to obtain sparse models, which } \\
\text { is important for large-scale problems because it reduces } \\
\text { storage space } \\
\begin{array}{l}\text { Elastic- } \\
\text { net }\end{array}\end{array}\|A(\omega)\|_{1},(1 / 2)\|A(\omega)\|_{2}^{2}$ & $\begin{array}{c}\text { Simple implementation } \\
\text { origin and requires special treatment }\end{array}$ \\
\hline
\end{tabular}

To validate the correctness and precision of EN in response prediction, EN is compared with OLS, and the method of transfer functions (TF) in which the known responses can be predicted by transfer functions and two independent excitation sources' load in this experiment. Moreover, these methods are compared in theory as shown in Table 2.

For the regularization method, which effectively mitigates the problem of having too large a number of conditions, the method is suitable for scenarios that do not require a lot of experimental data for training. However, many hyperparameters need to be tried.

2.6. The Application Scopes of Elastic-Net Regularization Based Multipoint Vibration Response Prediction in Frequency Domain under Uncorrelated Multiple Sources Load. The scope of application of the proposed method in this paper is as follows:

(1) The required data and the system need to be obtained under linear time-invariant conditions

(2) The locations and directions of the load and response points in the historical data are the same as those in the operating environment, and the $m$ loads are smoothly excited and uncorrelated with each other

(3) $K$ independent experiments must be conducted to find the linear relationship from load to response

(4) The proposed model is suitable for linear systems

\section{Experiment Verification}

3.1. Introduction of Experimental Object and Dataset. The experimental apparatus is a cylindrical structure fixed to the shaker table and suspended on an elastic rubber rope. In the interior of the cylindrical housing, there is an acoustic excitation device as shown in Figure 4 [19]. Externally, there is a vibration excitation of the vibration table which includes a sensor for recording the vibration excitation, an external acoustic excitation, and a vibration response sensor for the internal device. Figure 5 [19] shows the layout of the response measurement points, Figure 6 [19] shows the experimental environment, Figure 7 [19] shows the suspended vibration excitation source [19], and Figure 8 shows the layout of the vibration response measurement points.
TABLE 2: Comparison of multipoint response prediction methods.

\begin{tabular}{|c|c|c|c|}
\hline Model & $\begin{array}{l}\text { Transfer } \\
\text { function }\end{array}$ & $\begin{array}{l}\text { Multiple } \\
\text { linear } \\
\text { regression }\end{array}$ & $\begin{array}{l}\text { After elastic-net } \\
\text { regularization }\end{array}$ \\
\hline Indefinite problem & Yes & Yes & Solved. \\
\hline Transfer function & Yes & Yes & Not \\
\hline Robustness & Not & Not & Yes \\
\hline $\begin{array}{l}\text { Adaptation } \\
\text { systems }\end{array}$ & Linear & Linear & Linear \\
\hline $\begin{array}{l}\text { Historical training } \\
\text { datasets }\end{array}$ & Not & Yes & Yes \\
\hline Hyperparameter & Not & Not & A kind of \\
\hline Training time & Not & Short & Mid \\
\hline
\end{tabular}

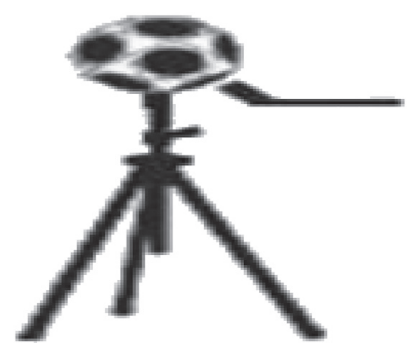

FIgURE 4: Spherical noise excitation source.

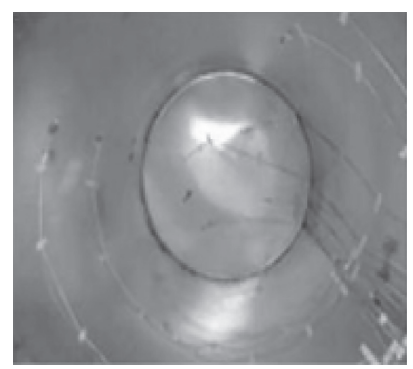

FIGURE 5: Cylindrical shell measurement nodes.

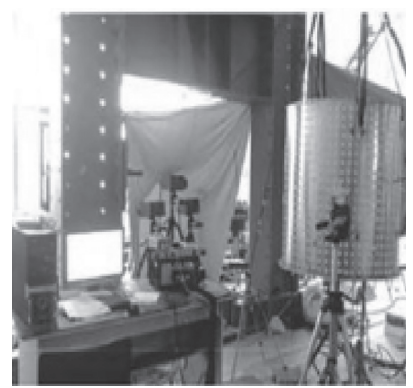

FIGURE 6: Experimental layout. 


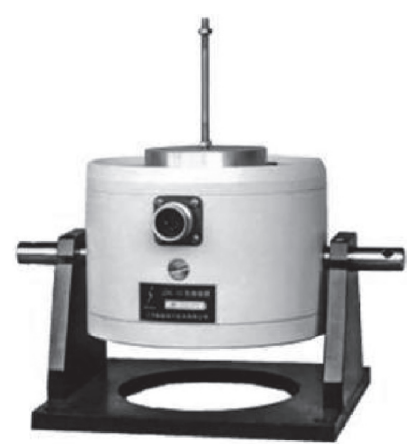

Figure 7: Suspended vibration excitation source.

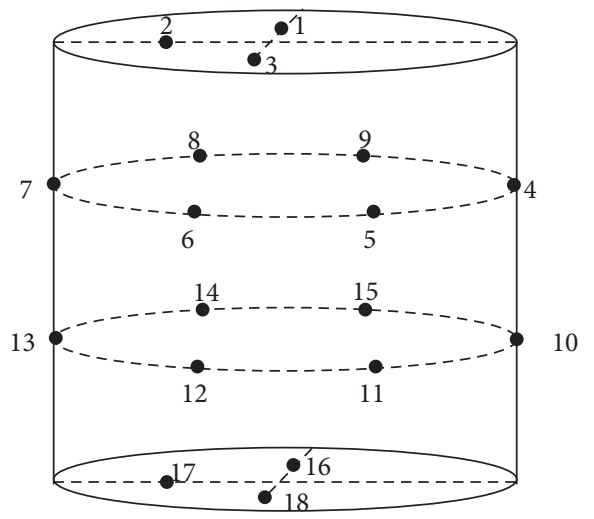

FIGURE 8: Location distribution of response measuring points.

\subsection{Experimental Design and Data Processing}

(1) Data Acquisition. In this experiment, there are two unrelated excitation sources: spherical noise excitation source and suspended vibration excitation source, and the number of unrelated excitation sources is $m=2$. The noise excitation device is divided into 3 magnitudes, and the vibration excitation device has 5 magnitudes, so there are 15 sets of unrelated multisource load excitation, and the data under all the collected working conditions are used as samples.

(2) Time-Frequency Domain Transformation. Because the discussion is on the frequency domain response prediction, first of all, the data from the time domain through the FFT transformation to the frequency domain, we can get the frequency data from $0 \mathrm{~Hz}$ to $6400 \mathrm{~Hz}$, frequency resolution of $4 \mathrm{~Hz}$, and then the frequency as a group based on the 1601 groups; each frequency corresponds to 15 groups of sample data.

(3) Regularized Hyperparameter Selection. A grid search was used to select final hyperparameters and choose the optimal hyperparameters of each of ridge regression, Lasso regression, and elastic network for the experiment and compare the experimental results.

\subsection{Evaluation Methods and Indicators}

3.3.1. Evaluation Methodology. Regarding training set and test set division, among the 15 sets of sample data under different working conditions, 14 sets of samples are used each time as historical training samples and 1 set of samples is used as test set samples. Cross-validation is performed by the leave-one-out method, and 15 rounds of experiments are conducted to obtain the statistical average results to evaluate the accuracy of the model.

3.3.2. Evaluation Indicator. $3 \mathrm{~dB}$ error rate is used to evaluate the model, which is a widely used evaluation tool in the process of engineering practice. The $3 \mathrm{~dB}$ relative error is shown in the following:

$$
10 \log _{10}\left|\frac{\widehat{S}_{y_{i} y_{i}}(\omega)}{S_{y_{i} y_{i}}(\omega)}\right| \leq 3 \text { and } 10 \log _{10}\left|\frac{S_{y_{i} y_{i}}(\omega)}{\widehat{S}_{y_{i} y_{i}}(\omega)}\right| \leq 3
$$

At frequency $\omega$, if the predicted response $\widehat{S}_{y_{i} y_{i}}(\omega)$ and the real load $S_{y_{i} y_{i}}(\omega)$ do not meet equation (8), the load is marked as $3 \mathrm{~dB}$ error load at frequency $\omega$, and the ratio of all load data that does not meet the standard to all predicted loads is $3 \mathrm{~dB}$ error rate.

In order to evaluate the prediction performance of the whole models, Root Mean Square Error (RMSE) is also used to evaluate the model. The formula is as follows:

$$
\mathrm{RMSE}=\sqrt{\frac{\sum_{i=1}^{\Omega}\left(S_{y_{i} y_{i}}(\omega)-\widehat{S}_{y_{i} y_{i}}(\omega)\right)^{2}}{\Omega}} .
$$

The smaller the RMSE is, the closer the predicted value is to the true value.

3.4. Experimental Parameter Setting. There are four hyperparameters in the experiments involved in this paper, as shown in Figures 9-11, and by selecting the RMSEs of different regularized hyperparameters for averaging and then using them as evaluation indexes, the optimal hyperparameters for the three regularization methods can be derived in Table 3 .

3.5. Experimental Results. To verify the correctness and accuracy of the method, the vibration response data from the unknown response measurement points estimated by applying the method need to be analyzed in comparison with the real vibration response data.

Table 4 gives the comparison of the results of the four methods after passing the leave-one-out method in the form of mean \pm standard deviation. Table 5 shows the comparison between preregularization and postregularization, shown in terms of the number of conditions and RMSE values.

In order to verify the effect of model training more visually, Figures 12 and 13 show the $3 \mathrm{~dB}$ error for response point 2 under two conditions (before $\mathrm{EN}$ and after $\mathrm{EN}$ ); 


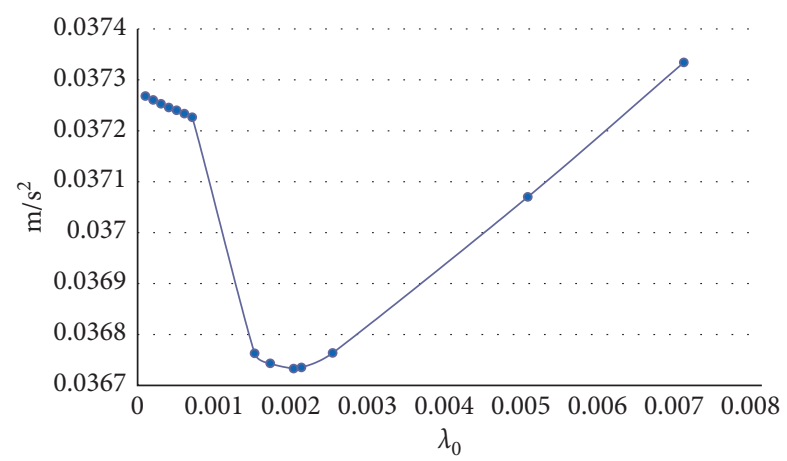

FIGURE 9: Graph for finding the Lasso optimal hyperparameters of the $8^{\text {th }}$ round.

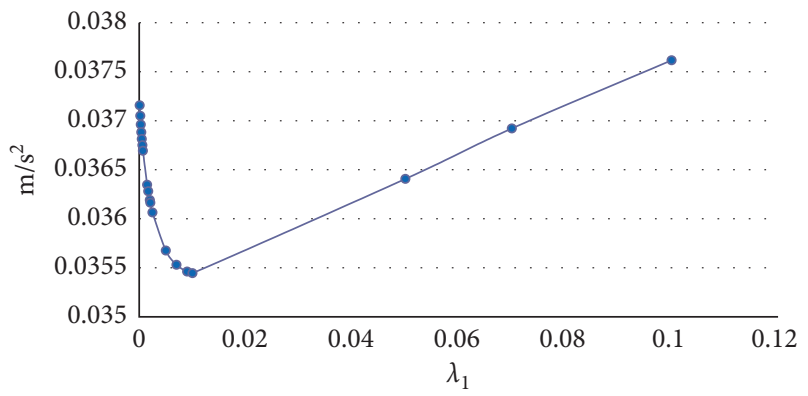

FIGURE 10: Graph for finding the ridge optimal hyperparameters of the $8^{\text {th }}$ round.

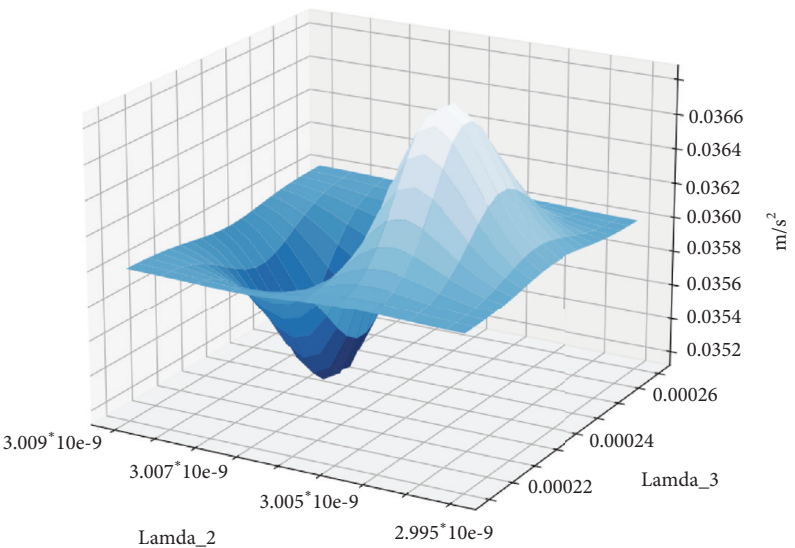

FigURE 11: Graph for finding the elastic-net optimal hyperparameters of the $8^{\text {th }}$ round.

TABLE 3: Detailed values of hyperparameters mean of 15 sets of experiments.

\begin{tabular}{lcr}
\hline Methods & Hyperparameters & Value \\
\hline Lasso & vdfdx & $1.351 * 10 e-2$ \\
Ridge & dvds & $2.113 * 10 e-3$ \\
Elastic-net & dvdsdg & $3.001 * 10 e-9$ \\
& dvds & $2 * 10 e-5$ \\
\hline
\end{tabular}


TABLE 4: $3 \mathrm{~dB}$ error results based on vibration response prediction model.

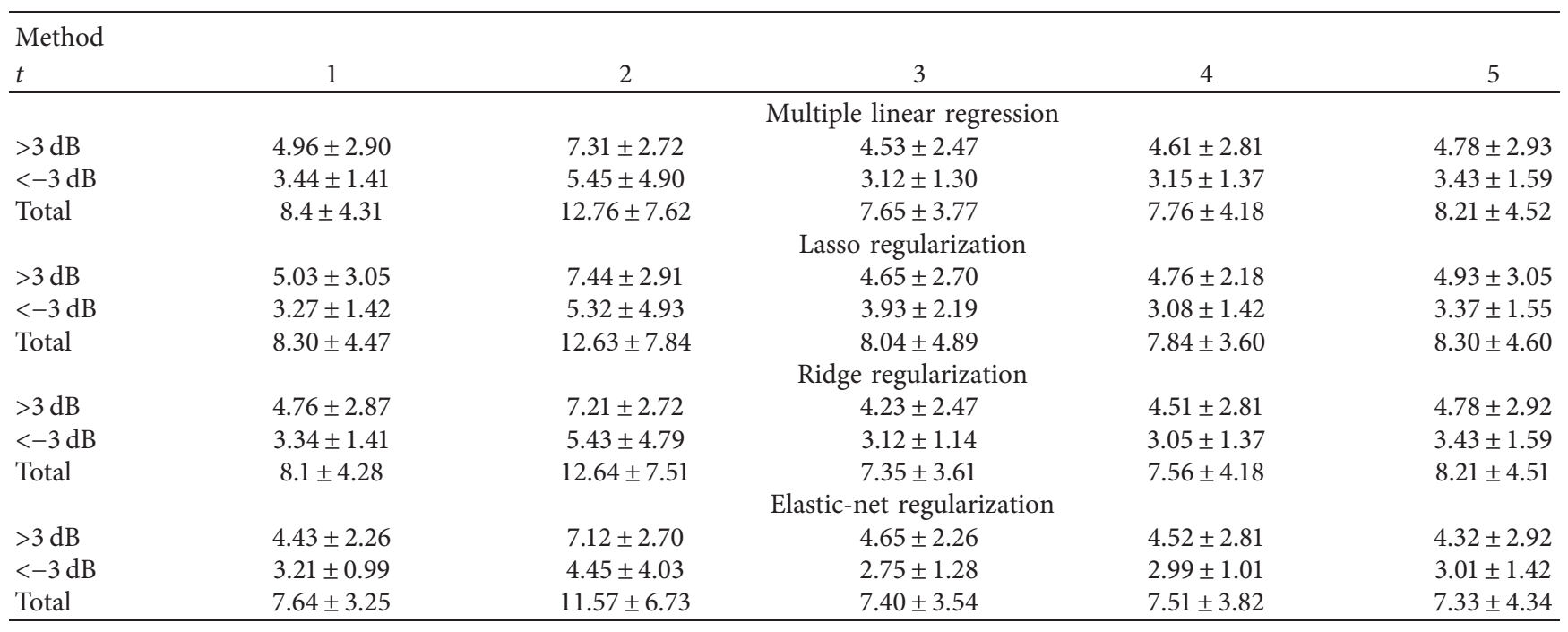

TABle 5: Preregularization and postregularization comparisons.

\begin{tabular}{lcccc}
\hline Inherent frequency $(\mathrm{Hz})$ & Conditions number & Conditions number after EN & RMSE & RMSE after EN \\
\hline 280 & 188.3 & 23 & 0.03526 & 0.01536 \\
536 & 98.9 & 8.8 & 0.03309 & 0.03051 \\
596 & 7.8 & 3.06 & 0.03025 & 0.02399 \\
1434 & 3.17 & 1.15 & 0.00966 & 0.00566 \\
1862 & 7.2 & 3.14 & 0.03230 & 0.02432 \\
\hline
\end{tabular}

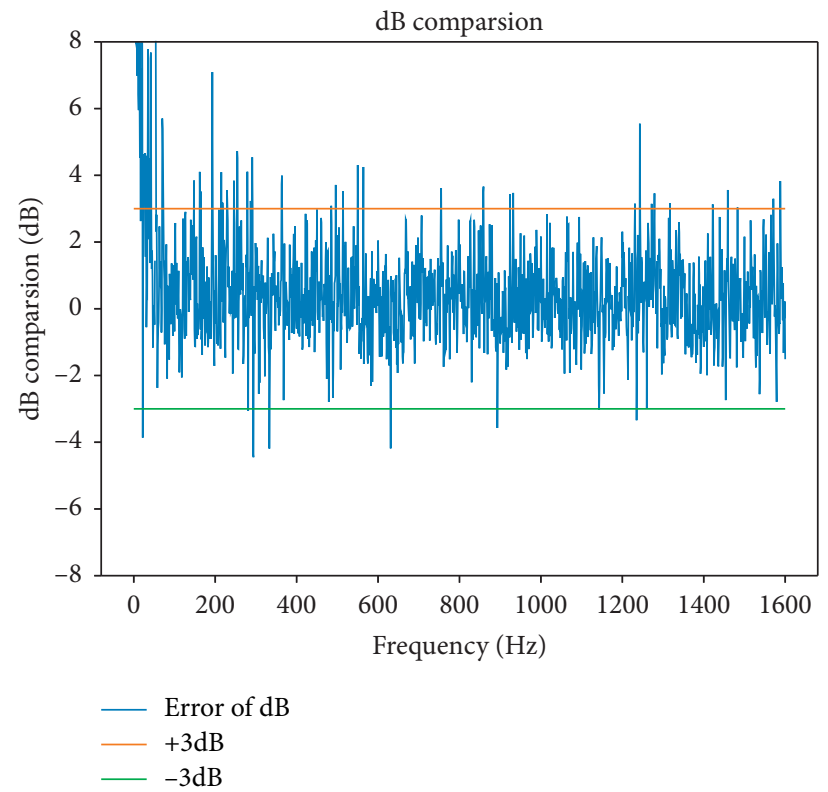

FIGURE 12: $3 \mathrm{~dB}$ error value for the $2^{\text {nd }}$ response measurement point of the $8^{\text {th }}$ round before elastic-net regularization. 


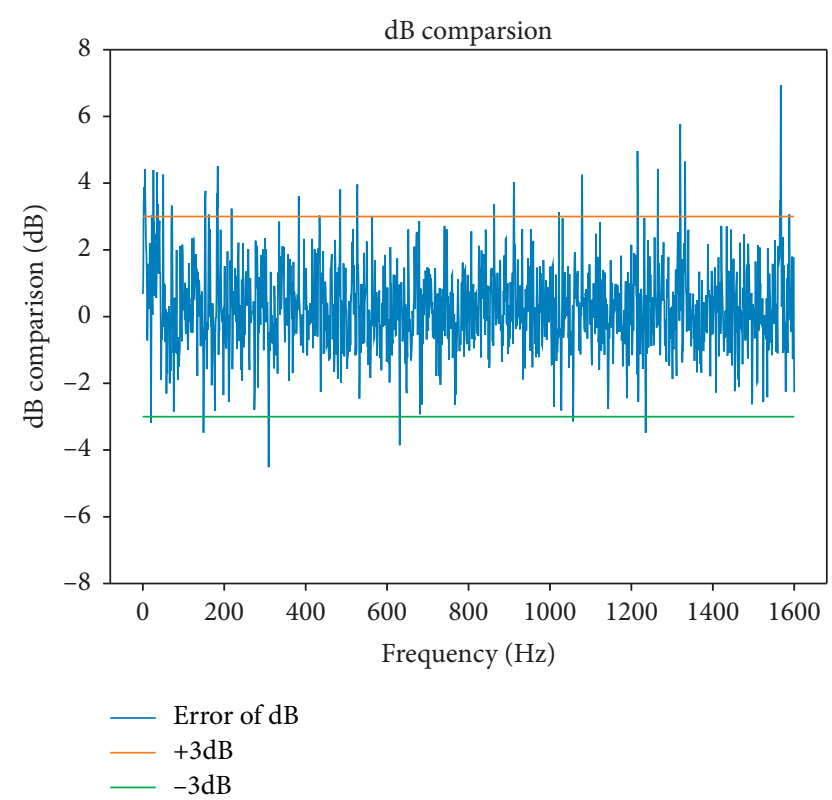

FIGURE 13: $3 \mathrm{~dB}$ error value for the $2^{\text {nd }}$ response measurement point of the $8^{\text {th }}$ round after elastic-net regularization.

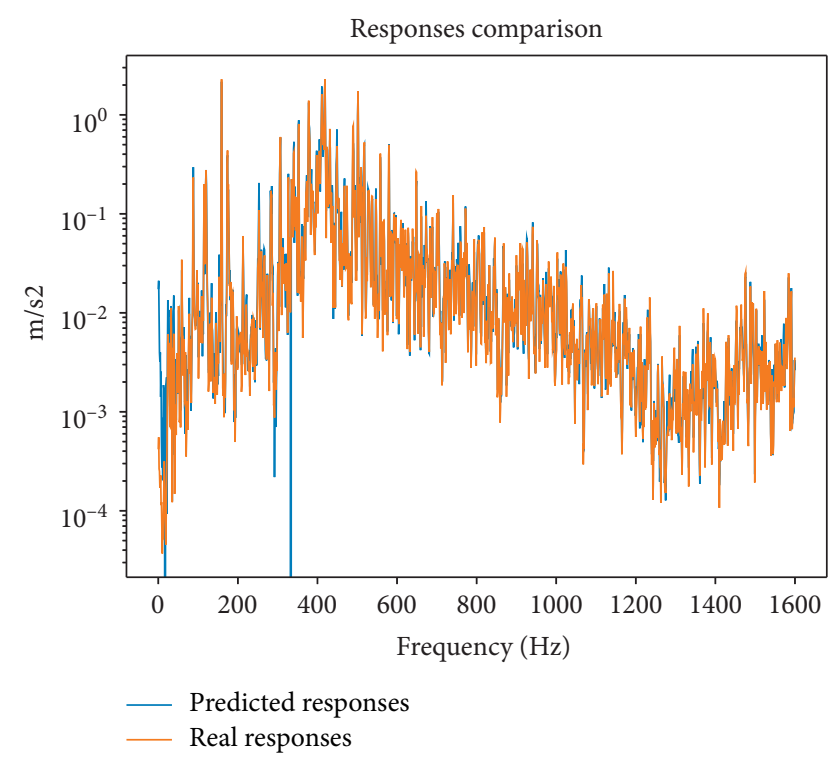

Figure 14: Comparison of predicted and real responses power spectra for the $2^{\text {nd }}$ response measurement point of the $8^{\text {th }}$ round before elastic-net regularization.

Figures 14 and 15 show the predicted response value and true response value for the first two response measurement points 2 under two conditions (before EN and after EN).

\subsection{Analysis of Experimental Results}

(1) From Table 4, it can be seen that the model trained by the elastic net has higher recognition accuracy than that using the multiple linear regression method and has better recognition accuracy compared to the other regularization methods (Lasso and ridge).

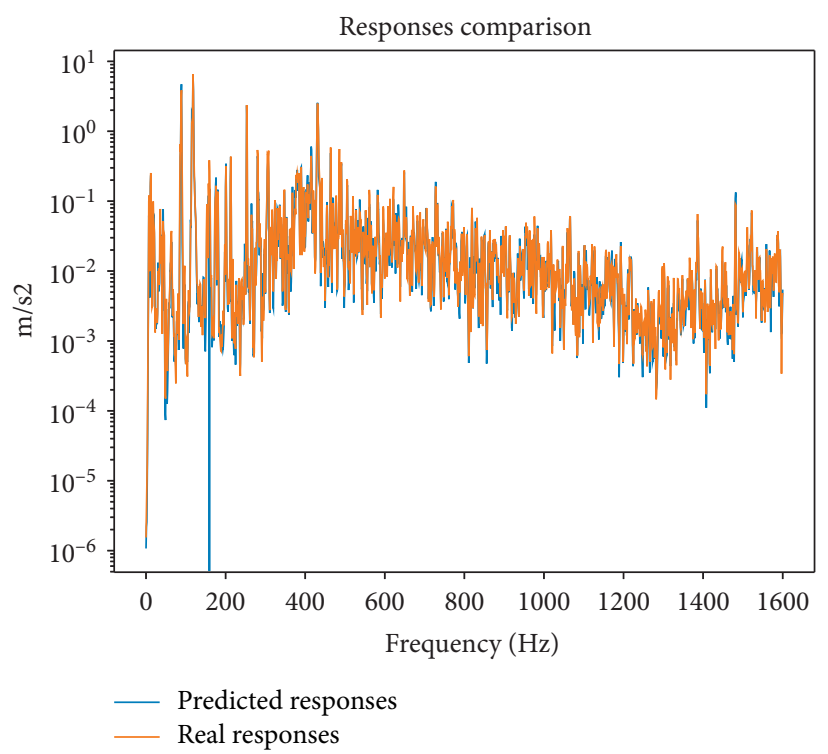

FIGURE 15: Comparison of predicted and real responses power spectra for the $2^{\text {nd }}$ response measurement point of the $8^{\text {th }}$ round after elastic-net regularization.

(2) However, multiple linear regression suffers from the following problems: Multiple linear regression also has the problem that the number of conditions is too large at some frequency points, as shown in Table 5. This problem is effectively solved by the improvement of regularization.

(3) From Figures 12 and 13, the $3 \mathrm{~dB}$ error value is from $5.05 \%$ to $3.86 \%$ for response point 2 after EN; it also can be seen that the improved model with the addition of regularization achieves a better result, and the predicted response is very close to the actual response, which basically meets the engineering requirements from Figures 14 and 15.

(4) To summarize the above analysis, the regularization can improve the prediction accuracy of the multiple regression prediction model, improve the training efficiency of the multipoint frequency domain vibration response prediction model based on multiple linear regression, and improve the model accuracy, and the model can meet the engineering requirements.

\section{Conclusion}

In order to solve the problems of large number of conditions at inherent frequencies and low prediction accuracy in the multiple multivariate linear regression vibration response prediction model, kinds of regularization methods were added to the multiple multivariate linear regression vibration response prediction model in order to alleviate the problem. Through the proposed method of this paper, the vibration response prediction technology can be applied to modern engineering design, reliability testing, vibration control, and so forth. By 
conducting effective and accurate vibration response prediction research for aerospace structures, bridges, buildings, and other constructions, significance has a certain guiding effect.

In this study, although the multivariate linear regression model was improved by regularization to alleviate the indeterminacy and matrix pathology problems of the model, at least one hyperparameter was introduced and additional hyperparameter research work was required. Therefore, in the next research program, a vibration response prediction method based on partial least-squares regression is proposed. The partial least-squares regression-based vibration response prediction method does not require hyperparameter research work. In addition, we also need to carry out further research on response prediction under nonlinear system conditions.

\section{Data Availability}

The data used to support the findings of this study are available from the first author upon request.

\section{Conflicts of Interest}

The authors declare that they have no conflicts of interest.

\section{Acknowledgments}

This work was financially supported by the National Natural Science Foundation of China (Grant nos. 51305142 and 51305143), the General Financial Grant from the China Postdoctoral Science Foundation (Grant no. 2014M552429), and project of Quanzhou Science and Technology Plan (Grant no. 2018C110R and 2018C114R). Research works in this paper are also supported by the National Key Technology R\&D Program of China (Grant no. 2018YFB1402500), the Social Science Planning Foundation of Fujian Province (Grant no. FJ2020B0033), and the Scientific Research Funds of Huaqiao University (Grant no. 16BS304).

\section{References}

[1] W. Liu, M. Ma, and B. Wangwen, "Prediction of environmental response of metro train vibration," China Railway Science, vol. 34, no. 4, pp. 110-117, 2013.

[2] F. Li and Q. Du, "Prediction of turntable vibration response based on frequency response function," Noise and Vibration Control, vol. 35, no. 3, pp. 121-123, 2015.

[3] C. Zhuchang, L. Wangmao, and M. Zengqi, "Prediction of vibration environmental test response based on vibration transmission characteristics," Journal of Southwest Jiaotong University, vol. 37, no. B11, pp. 1-4, 2002.

[4] M. Xuzhang, M. Gaotian, and Y. Shenrong, "An improved method of finite element model correction using frequency response function," Vibration and Impact, vol. 21, no. 3, pp. 43-45, 2002.

[5] F. Li and Q. Du, "Prediction of turntable vibration response based on frequency," Noise and Vibration, vol. 35, pp. 121123, 2015.
[6] F. John, "Force estimation using operational data," in Proceedings of 8th International Modal Analysis Conference, pp. 1586-1592, SPIE, Copenhagen, Denmark, 1990.

[7] M. Maoyu, F. Linjian, and H. Liujing, "Recent advances of dynamic force estimation techniques"," Journal of Dynamics and Control, vol. 12, no. 2, pp. 97-102, 2014.

[8] Y. Liu, "Mathematical model of multivariate linear regression"," Journal of Shenyang Engineering College (Natural Science Edition), vol. 1, no. 2, pp. 128-129, 2005.

[9] W. Wanghui and J. Meng, "Predictive modeling by multiple linear regression,", Journal of Beijing University of Aeronautics and Astronautics, vol. 33, no. 44, pp. 500-504, 2007.

[10] S. Fanshan, "Application of linear regression model for land subsidence prediction in Beijing plain," The Chinese Journal of Geological Hazard and Control, vol. 24, no. 1, pp. 70-74, 2013.

[11] H. Jin and Y. Gaosu, "Selection of the optimal multiple linear regression model," Journal of Hebei University of Technology, vol. 31, no. 5, pp. 10-14, 2002.

[12] P. Peng and H. Pengjia, "Research on power load prediction based on multiple linear regression model"," China Safety Production Science and Technology, vol. 7, no. 9, pp. 158-161, 2011.

[13] J. Liujin, The Regularization Method and Application of the Non-definite Problem, Science Press, Beijing, China, 2005.

[14] Q. Huyu, "Regularization parameter selection method based on parsing,"," Journal of Jinling Institute of Science and Technology, vol. 35, no. 4, pp. 61-65, 2019.

[15] Chitra, "Fractional Tikhonov regularization method in Hilbert scales," Applied Mathematics and Computation, vol. 392, 2021.

[16] Y. Wuyang, "Nonstationary signal inversion based on shaping regularization for random noise attenuation," Applied Geophysics, vol. 3, p. 17, 2021.

[17] C. Wang, M. Laixiong, and J. Jiao, "Load identification of acoustic and vibration sources following linear regression and least-squares of generalized matrix inverse method," Journal of Information and Computational Science, vol. 11, no. 9, pp. 3229-3239, 2014.

[18] A. E. Hoerl and R. W. Kennard, "Ridge regression: biased estimation for nonorthogonal problems," Technometrics, vol. 12 , no. 1, pp. 55-67, 1970.

[19] W. Zhan, "Multipoint vibration response prediction method based on principal component linear regression under multisource unknown load conditions," Computer Integrated Manufacturing Systems, vol. 25, no. 3, pp. 577-585, 2019. 